\title{
Medicine in the Elderly
}

\section{Anorexia nervosa in a 67 year old woman}

\author{
M.D. Ramell and N. Brown ${ }^{1}$
}

Digby Hospital, Woodwater Lane, Exeter EX27EY, Devon and ${ }^{1}$ Royal Devon and Exeter Hospital (Wonford), Exeter, Devon, UK.

\begin{abstract}
Summary: The case is described of anorexia nervosa arising in a 62 year old woman with symptoms persisting over the following 5 years.
\end{abstract}

\section{Introduction}

Anorexia nervosa is characterized by a dramatic weight loss caused by the patient's steadfast reluctance to eat an adequate diet, often related to a disturbance of body image and an exaggerated fear of becoming obese. Although it typically occurs in adolescent females, cases with onset later in life have been reported and we believe this case to be the oldest yet described.

\section{Case report}

The patient presented initially to her general practitioner with multiple physical complaints, including pruritus, gingivitis, and poor peripheral circulation, probably related to dietary deficiency. She was subsequently referred for psychiatric assessment of her eating habits. Her symptoms began aged 62 when, shortly following her retirement from assisting in a patisserie, she ate rather heavily over Christmas and her weight increased from her usual $53 \mathrm{~kg}$ to $61 \mathrm{~kg}$. She returned to her usual weight after 1 month's dieting, but was not satisfied at this, and despite reassurance from an obesity clinic continued to diet until her weight fell to about $40 \mathrm{~kg}$ which she maintained for 4 years.

She showed a total preoccupation about her weight and calorie intake $(400-600 \mathrm{kcal} /$ day $)$ which she recorded in detail. She always felt hungry, had a good appetite, and enjoyed a varied diet but if a normal sized meal was prepared for her (e.g. when visiting her daughter) she would feel obliged to eat

Correspondence: M.D. Ramell, M.B., B.S.

Accepted: 5 August 1987 it, then later induce vomiting by putting her fingers in her throat. She did not binge food, nor did she abuse laxatives, using no more than two Senokot tablets at night if required to regulate her bowel habit, as she had done for many years.

She had an intense fear of becoming obese, with distortion of her body image. She felt fat but at the same time agreed that her weight was belowo average. However strictly she controlled her intake, she could never lose enough weight to relieve her sense of obesity. On an eating attitude test she scored 68 where a value of above 30 is regarded as diagnostic. ${ }^{1}$ She was motivated by the desire to 'look nice', but had considerable anxiety and guilt about her anorexic behaviour.

When aged 34 she had a colostomy following an abdominoperineal bowel resection, hysterectomy and bilateral oophorectomy for endometriosis. She was self-conscious of the colostomy, and managed without a bag.

Seven years prior to the onset of her anorexic habits she had suffered three major losses: that of her sister during an asthmatic attack, resulting in the suicide of her severely arthritic father two months later, and finally her husband with bronchial carcinoma. She felt that she had not been able to grieve fully and had suffered episodic depression since then, treated with tricyclic antidepressants and support from her general practitioner. There was no other personal or family psychiatric history.

She was treated initially with group therapy, relaxation, antidepressants and tranquillizers for 5 years with no real improvement. She was admitted at the age of 67 to a general medical ward. On admission she was $150 \mathrm{~cm}$ in height, and $41 \mathrm{~kg}$ in

(C) The Fellowship of Postgraduate Medicine, 1988 
weight, thus $26 \%$ below her optimal weight. She had dry waxy skin and a smooth tongue but apart from her healthy colostomy physical examination was unremarkable.

Liver and thyroid function tests, blood urea and electrolytes were all within normal limits, as were the serum carotene, cholesterol, glucose, prolactin, $B_{12}$ and folate concentrations. The full blood count was unremarkable and the ESR was $1 \mathrm{~mm} / \mathrm{h}$. A butterfat absorption test was normal. According to local practice on the medical ward she was treated with small doses of chloropromazine and insulin with a free choice of diet, and rapidly put on weight with daily monitoring. She was discouraged in the use of stimulant laxatives, regular colostomy actions being promoted using bulk preparations alone. After $3 \frac{1}{2}$ weeks her weight had increased by $7 \mathrm{~kg}$ to $48 \mathrm{~kg}$ and she felt more at ease with her increased food intake. She was considered to be well enough for discharge on a small dose of chlorpromazine only, and, apart from a short relapse following a minor road traffic accident, had maintained her improvement 6 months after discharge. When last seen she was less preoccupied with calorie counting and her weight was $48.5 \mathrm{~kg}$.

\section{Discussion}

This patient displayed many of the diagnostic features of anorexia nervosa. She had marked weight loss and disturbance of body image, together with a great fear of becoming obese and a constant preoccupation with measuring and regulating food

\section{References}

1. Garner, D.M. \& Garfinkel, P.E. The Eating Attitudes Test: an index of the systems of anorexia nervosa. Psychol Med 1979, 9: 273-279.

2. Ryle, J.A. Anorexia nervosa. Lancet 1936, ii: 893-899.

3. Kellett, J., Trimble, M. \& Thorley, A. Anorexia nervosa after the menopause. Br J Psychiatry 1976, 128: $555-558$. intake. The criteria of amenorrhoea could not, of course, be applied as she had been post-menopausal for 30 years. Although she would induce vomiting if she had been obliged by social pressures to eat what she considered an excessive amount, there was no regular bingeing or purging behaviour. In view of her major abdominal surgery it was important to exclude any physical condition accounting for her weight loss. The pathology in 1952 was histologically proven, and there was no evidence of recurrence or malabsorption. Furthermore she had rapid weight gain as an hospital in-patient.

Whilst most published series suggest a mean age of onset of about 17 years, cases with older onset have been reported. Ryle ${ }^{2}$ described 13 patients with onset over the age of 30 , the eldest being 59 , but provided poor diagnostic criteria. The first detailed account of true post-menopausal anorexia nervosa describes onset in a 52 year old woman. ${ }^{3}$ Bernstein $^{4}$ describes a 94 year old woman with features of the condition related to a depressive illness which would qualify as secondary anorexia nervosa as classified by King. ${ }^{5}$

Our patient has thus suffered from anorexia nervosa for the last 5 years since the diagnosis was made aged 62. Although mainly a condition of adolescence it can develop in patients of any age.

\section{Acknowledgements}

We would like to thank Dr S. Bhanji and Professor D. Mattingly for permission to publish their clinical material.

4. Bernstein, I.C. Anorexia nervosa: 94 year old lady treated with electric shock. Minn Med 1972, 55: 552553.

5. King, A. Primary and secondary anorexia nervosa syndromes. Br J Psychiatry 1963, 109: 470-479. 